\title{
Functional analysis of the flagellar genes in the fliD operon of Salmonella typhimurium
}

\author{
Tatsuki Yokoseki, ${ }^{1}$ Kazuhiro Kutsukake, ${ }^{1}$ Kouhei Ohnishi ${ }^{2} \dagger$ \\ and Tetsuo lino ${ }^{2}$
}

Author for correspondence: Kazuhiro Kutsukake. Tel: +81 82424 7924. Fax: +81 824227067.

\footnotetext{
${ }^{1}$ Faculty of Applied Biological Science, Hiroshima University, Kagamiyama 1-4-4, Higashi-Hiroshima, Hiroshima 739, Japan

2 School of Human Sciences, Waseda University, Tokorozawa, Saitama 359, Japan
}

\begin{abstract}
The fliD genes of Salmonella typhimurium and Escherichia coli encode the filament-cap protein of the flagellar apparatus, which facilitates the polymerization of endogenous flagellin at the tips of the growing filaments. Previous sequence analysis of this operon in both organisms has revealed that the fliD gene constitutes an operon together with two additional genes, flis and fiiT. Based on the gene-disruption experiment in E. coli, both the flis and flit genes have been postulated to be necessary for flagellation. In the present study, we constructed S. typhimurium mutants in which either flis or flit on the chromosome was specifically disrupted. Both mutants were found to produce functional flagella, indicating that these genes are dispensable for motility development in S. typhimurium. However, flagellar filaments produced by the flis mutant were much shorter than those produced by the wild-type strain. This indicates that the flis mutation affects the elongation step of filament assembly. The excretion efficiency of flagellin was examined in the fliD-mutant background, where the exported flagellin molecules cannot assemble onto the hooks, resulting in their excretion into the culture media. We found that the amount of flagellin excreted was much reduced by the flis mutation. Based on these results, we conclude that Flis facilitates the export of flagellin through the flagellum-specific export pathway.
\end{abstract}

Keywords: Salmonella, flagellar gene, filament elongation, gene disruption, flagellumspecific export pathway

\section{INTRODUCTION}

The flagellum of Salmonella typhimurium and Escherichia coli is a locomotive organelle composed of three structural parts : a basal body, a hook and a filament (Macnab, 1992). The filament extends into the extracellular space and is connected by the hook to the basal body embedded in the cell membrane. Flagellar assembly proceeds from the cellproximal structure to the cell-distal structure. Assembly of the extracellular structures (filament and hook) is believed to involve transport of the component proteins through the flagellum-specific transport pathway, which is supposed to reside within the flagellar structure. The filament consists of a single kind of protein, flagellin. Polymerization of flagellin onto the tips of the hook requires three hook-associated proteins, FlgK, FlgL and

†Present address: Department of Molecular Biophysics and Biochemistry, Yale University, New Haven, CT 06520-8114, USA.
FliD (Homma et al., 1984b, 1986). FlgK and FlgL exist at the tip of the hook constituting the hook-filament junction layer, which acts as a polymerization nucleus for flagellin monomers (Homma \& Iino, 1985; Ikeda et al., 1987, 1989). FliD acts as a capping protein of the filament, and is essential for polymerization of newly exported flagellin monomers at the tips of the growing filaments (Ikeda et al., 1985, 1993; Homma et al., 1986).

So far, nearly 50 genes have been shown to be involved in flagellar formation and function (Macnab, 1992). Almost all of them are clustered in four regions on the chromosome, called regions I, II, IIIa and IIIb. The $f g K$ and $f g L$ genes together constitute an operon in region $I$, while the $f i D$ gene and the flagellin gene, $f i C$, form independent operons in region IIIa (Kutsukake et al., 1988). On the basis of the sequence analysis of the fiD operons from $E$. coli and S. typhimurium, Kawagishi et al. (1992) showed that the fiD gene constitutes an operon together with two additional genes, $f i S$ and $f i T$, which encodes $15 \mathrm{kDa}$ and $14 \mathrm{kDa}$ proteins, respectively. They constructed an E. coli 
mutant, IK23, in which the chromosomal fiDST region was deleted. Based on the complementation pattern of IK23 with the recombinant plasmids carrying various parts of the $f i D$ operon, they concluded that the $f i S$ and fiT genes are both indispensable for flagellation in E. coli. Recently, Chen \& Helmann (1994) identified Bacillus subtilis genes homologous to $f i D, f i S$ and $f i T$ which are also likely to constitute an operon. They showed that integration of a plasmid into the chromosomal fiS gene led to a $\mathrm{Fla}^{-}$phenotype, and concluded that at least the fiS gene is indispensable for flagellation in B. subtilis.

This work was initiated to elucidate the function of the genes in the $f i D$ operon of $S$. typhimurium in flagellar morphogenesis. For understanding the function of specific genes, it is important to isolate defined mutants defective in the genes. Using the gene-replacement method developed by Yamada et al. (1993), we constructed mutants in which the chromosomal fiS and $f i T$ genes were specifically disrupted. To our surprise, both fiS and fiT mutants were found to produce functional flagella, indicating that these genes are dispensable for flagellation. However, the fis mutant produced flagella with short filaments, suggesting that the fis product is required for efficient elongation of the filament. The amount of flagellin excreted from the cells was found to be much reduced by the fliS mutation in the fiD-mutant background. Therefore, we conclude that FliS facilitates the export of flagellin through the flagellum-specific export pathway.

\section{METHODS}

Bacterial strains, plasmids and media. Strains used in the present study are all derivatives of an $S$. typhimurium wild-type strain, KK1004 (Kutsukake et al., 1988). KK2601 and KK2604 carry $f i D:: \operatorname{Tn} 10$ and $f i C:: \operatorname{Tn} 10$ mutations, respectively (Kutsukake et al., 1988). The procedure for construction of gene-disruption mutants in the $f i D$ operon is described below. Plasmids used were pKKD2 and pKKD4, both of which have been derived from a plasmid vector, pHSG398 (Takeshita et al., 1987). pKKD2 carries a $5.9 \mathrm{~kb}$ SalI fragment of the $S$. typhimurium chromosome inserted into the $\mathrm{SalI}$ site of pHSG398. This fragment contains both the $f i C$ and $f i D$ operons. pKKD4 carries a $1.9 \mathrm{~kb}$ Pst I-HindIII fragment of the $S$. typhimurium chromosome inserted into the Pst $\mathrm{I}-\mathrm{H}$ indIII site of pHSG398. This fragment contains only the $f i D$ gene. Plasmid pUC4K, carrying a kan gene cassette, was obtained from Pharmacia. Ordinary culture media including L broth, L-broth agar and motility agar plates were made as described previously (Kutsukake et al., 1988). Minimal medium for assay of excretion of flagellin was made as described previously (Kutsukake, 1994). Antibiotics were used at a final concentration of $25 \mu \mathrm{g} \mathrm{ml}^{-1}$.

Motility assay. Motile phenotype of cells was detected as formation of spreading colonies (swarms) on motility agar plates at $37^{\circ} \mathrm{C}$.

DNA manipulation. Restriction enzymes and DNA-modifying enzymes were purchased from Toyobo and Nippon Gene. Procedures for DNA manipulation and transformation were as described previously (Kutsukake et al., 1985). DNA sequencing was performed by the dideoxy chain-termination method

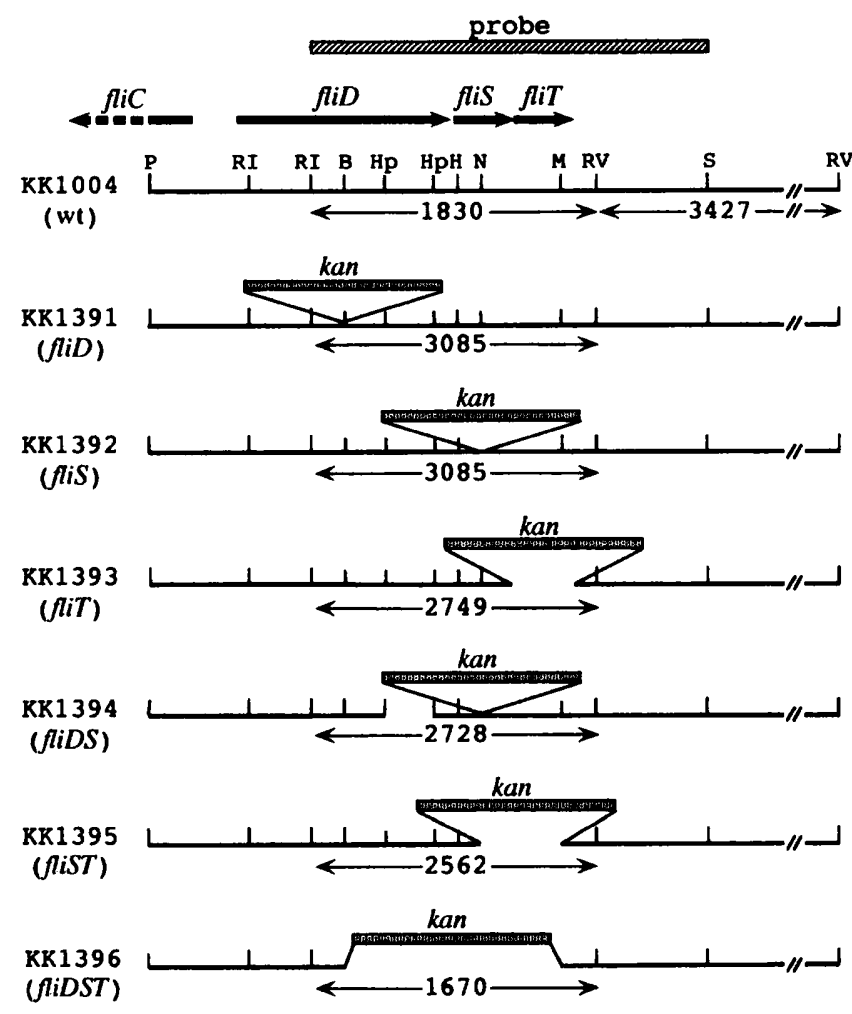

$1000 \mathrm{bp}$

Fig. 1. Structure of the chromosomal region containing the fliD operon in the wild-type and mutant strains of S. typhimurium. Procedures for construction of the mutant strains are described in the text. Restriction sites were adopted from the nucleotide sequence data reported by Kawagishi et al. (1992) and are shown using the following abbreviations: B, Bsu36l; RI, EcoRI; RV, ECoRV; H, HindlII; Hp, Hpal; M, Mlul; N, Nael; P, Pstl; S, Sall. Arrows indicate the coding regions for the individual genes. kan indicates the kan gene cassette. The extent of the probe DNA used in Southern blot analysis (Fig. 2) is indicated by the hatched bar. The length of the DNA fragment which should be detected by Southern blot analysis is shown in bp under the chromosomal structure of each strain.

(Sanger et al., 1977) with a Sequenase Version 2.0 sequencing kit (USB). Restriction fragments were purified from agarose gels with a Geneclean II kit (Bio101). Electroporation of $S$. typhimurium cells was performed using a Gene Pulsar system (Bio-Rad). Southern hybridization analysis of chromosomal DNA was carried out with a DIG DNA labelling and detection kit (Boehringer Mannheim) according to the manufacturer's recommendation.

Plasmid construction. The structure of the $f i D$ operon and its adjacent region on the $S$. typhimurium chromosome is summarized in Fig. 1. The $f i D$, fliS and $f i T$ genes encode proteins of 467, 135 and 122 amino acids, respectively. Plasmid pKKD2 has unique Bsu36I and $N a e I$ sites at the 248 th codon of the $f i D$ gene and at the $72 \mathrm{nd}$ codon of the fiiS gene, respectively. This plasmid was digested with Bsu36I, blunt-ended with T4 DNA polymerase, and ligated with the kan gene cassette which had been excised from pUC4K with SalI and blunt-ended with T4 DNA polymerase. The resulting hybrid plasmid was designated pKKD2D. The same kan gene cassette was inserted into a NaeI- 
digested pKKD2 to obtain pKKD2S. pKKD2 has a unique $M l u \mathrm{I}$ site at the 105 th codon of the $f i T$ gene. This plasmid was digested with $M l u$ I and treated with exonuclease III. After treatment with mung bean nuclease and Klenow enzyme, the plasmid DNA was ligated with the kan gene cassette which had been excised from pUC4K with EcoRI and blund-ended. Sequence analysis revealed that in the resulting plasmid the entire coding region of $f i T$ had been deleted and replaced with the kan gene cassette but the fiS gene remained intact. This plasmid was designated pKKD2T. pKKD2, which contains two HpaI sites within the fiD gene, was digested with $H p a I$ and self-ligated to obtain $\mathrm{pKKD} 2 \mathrm{H}$. The $f i D$ gene on this plasmid encodes a FliD protein lacking $175 \mathrm{C}$-terminal amino acids. The kan gene cassette was inserted into $\mathrm{pKKD} 2 \mathrm{H}$ at the $\mathrm{Nael}$ site to obtain pKKD2HDS. pKKD2 was digested with $N a e I$ and $M l u I$ or Bsu36I and MluI, blunt-ended with T4 DNA polymerase, and ligated with the kan gene cassettes which had been excised from pUC4K with PstI and blunt-ended with T4 DNA polymerase. The resulting hybrid plasmids were designated pKKD2ST or pKKD2DST, respectively. In pKKD2ST, 64 C-terminal codons of the $f i S$ gene and $104 \mathrm{~N}$-terminal codons of the $f i T$ gene have been removed. In pKKD2DST, $225 \mathrm{C}$-terminal codons of the $f i D$ gene, an entire $f i S$ gene and $104 \mathrm{~N}$-terminal codons of the $f i T$ gene have been removed.

Gene disruption. The genes on the chromosome were replaced with artificially disrupted genes on the plasmids according to the preligation method developed by Yamada et al. (1993). The detailed procedure is described in the Results section.

Preparation of flagellin monomers and in vitro reconstruction of filaments. Monomeric flagellin was purified from the wild-type and $f i S$-mutant strains by the method described by Asakura et al. (1964). For reconstruction of flagellar filaments onto the hooks of fiD-mutant cells, the method described by Kagawa et al. $(1981,1983)$ was adopted. The purified flagellin monomers were added to cells suspended in $\mathrm{PBS}(0 \cdot 145 \mathrm{M} \mathrm{NaCl}$; $0 \cdot 15 \mathrm{M}$ sodium phosphate) and incubated at $26^{\circ} \mathrm{C}$ for $12 \mathrm{~h}$. Formation of filaments was examined by electron microscopy.

Electron microscopic observation of flagella. In order to examine the flagellar structures attached to the cell bodies, cells grown in $\mathrm{L}$ broth were negatively stained with $1 \%(\mathrm{w} / \mathrm{v})$ phosphotungstic acid ( $\mathrm{pH} 7)$ and observed with a JEM120EX electron microscope (JEOL). In order to examine the flagellar basal structures, flagella including the hook-basal-body structures were fractionated from the cells by the method of Suzuki et al. (1978). The fractionated materials were negatively stained and observed by electron microscopy.

Electrophoretic analysis of flagellin. For the analysis of flagellin excreted into the culture medium, the cells were grown at $37^{\circ} \mathrm{C}$ in the minimal medium and aliquots of culture containing a constant number of cells were clarified by centrifugation. Proteins in the resulting supernatants were precipitated by $10 \%(\mathrm{v} / \mathrm{v})$ TCA and suspended in sample loading buffer $(50 \mathrm{mM}$ Tris/ $\mathrm{HCl}, \mathrm{pH} \mathrm{6.8}, 100 \mathrm{mM}$ dithiothreitol, $2 \%$, w/v, SDS, $0 \cdot 1 \%$ bromophenol blue, $10 \%$, v/v, glycerol) containing saturated Tris base. Samples were heated at $100{ }^{\circ} \mathrm{C}$ for $3 \mathrm{~min}$ and separated on polyacrylamide gels. The gels were stained with Coomassie brilliant blue R250. For the analysis of total flagellin, aliquots of culture containing a constant number of cells were mixed with the sample loading buffer and heated as above. After SDS-PAGE, the proteins on the gel were transferred onto a nitrocellulose membrane and incubated with a polyclonal rabbit antibody against flagellin. The blot was developed with a Smilight Western blotting kit (Sumitomo).

\section{RESULTS}

\section{Construction of gene-disruption mutants in the fliD operon}

Plasmids pKKD2D, pKKD2S and pKKD2T carry the kan gene cassettes inserted into the $f i D$, fii and $f i T$ genes, respectively. From these plasmids, the fragments which contain the disrupted genes but not the replication machinery were excised with PvuII and self-ligated. The resulting circular molecules were introduced into a wildtype strain, KK1004, by electroporation, and kanamycinresistant transformants were selected. The chromosomal DNAs were isolated from these transformants and digested with EcoRI and EcoRV simultaneously. The digested samples were separated by agarose-gel electrophoresis and analysed by Southern blotting using as a probe the 2574 bp EcoRI-SalI fragment from pKKD2 (Figs 1 and 2). The $1830 \mathrm{bp}$ and $3427 \mathrm{bp}$ fragments should be detected in the chromosomal DNA from KK1004. On the other hand, in the chromosomal DNAs whose fiD, $f i S$ or $f i T$ genes were replaced with the disrupted ones from the plasmids, the $1830 \mathrm{bp}$ fragment should be replaced by fragments of various lengths (Fig. 1). Transformants which showed the expected chromosomal structures were saved and used for further experiments. The strains in which the $f i D, f i S$ and $f i T$ genes on the chromosome were specifically disrupted were designated KK1391, KK1392 and KK1393, respectively. According to the same procedure, we constructed strains in which the chromosomal $f i D$ operons were replaced with the corresponding DNAs from pKKD2DS, pKKD2ST and pKKD2DST. Their chromosomal structures were also confirmed by Southern blotting (Fig. 2). They were designated KK1394, KK1395 and KK1396, respectively.

\section{Motility of the gene-disruption mutants}

Kawagishi et al. (1992) reported that all the genes in the $f i D$ operons are indispensable for flagellation in E. coli. If this was also the case in $S$. typhimurium, we could expect

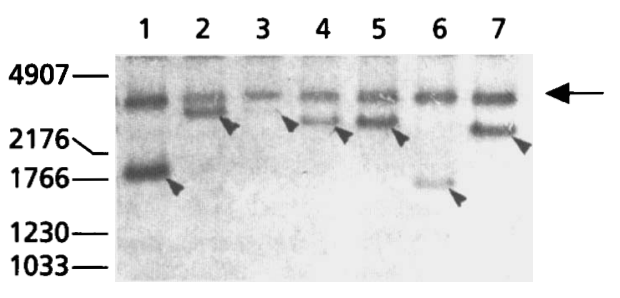

Fig. 2. Southern blot analysis of the chromosomal DNAs from the wild-type and mutant strains (Fig. 1). Chromosomal DNAs were digested with ECORI and ECORV simultaneously and separated by agarose-gel electrophoresis. Southern blotting was carried out using the $2574 \mathrm{bp}$ EcoRl-Sall fragment as a hybridization probe (Fig. 1). The position of the 3427 bp EcoRV fragment which should be detected in all the DNA samples is indicated by the arrow on the right. The arrowheads indicate the EcoRI-ECORV fragments within which the kan gene cassettes were inserted. The positions of the molecular length markers are indicated on the left in bp. Chromosomal DNAs used are as follows. Lanes: 1, KK1004; 2, KK1391; 3, KK1392; 4, KK1393; 5, KK1394; 6, KK1396; 7, KK1395. 


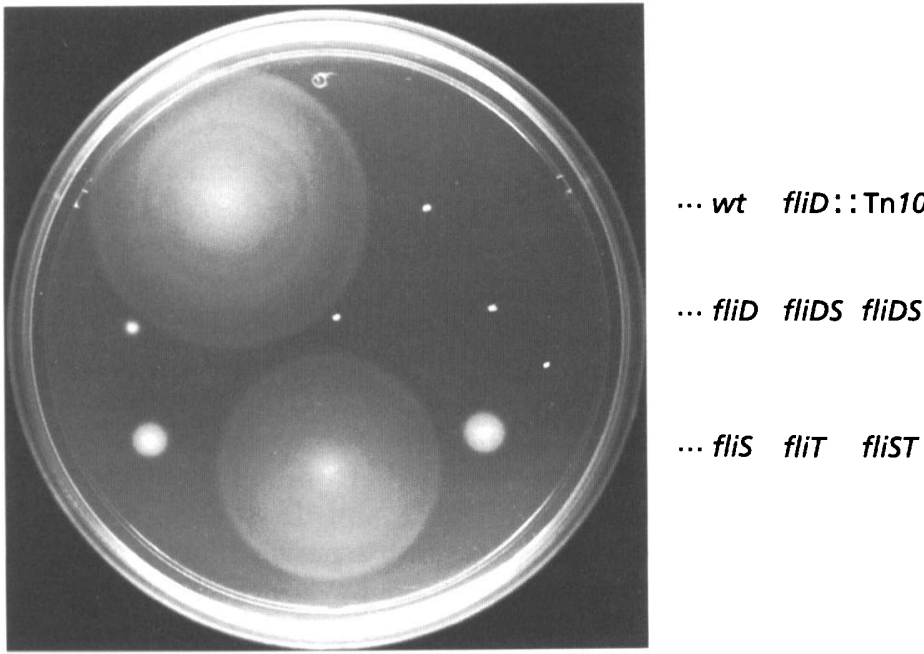

Fig. 3. Motile phenotypes of the wild-type and mutant strains. Single colonies formed on the L-broth agar plates were stabbed onto a motility agar plate and incubated for $5 \mathrm{~h}$ at $37^{\circ} \mathrm{C}$. Strains used are indicated on the right and as follows: wild-type, KK1004; fliD::Tn10 mutant, KK2601; fliD mutant, KK1391; fliDS mutant, KK1394; fliDST mutant, KK1396; flis mutant, KK1392; fliT mutant, KK1393; flisT mutant, KK1395.

that none of the disruption mutants constructed above were motile. In order to test this possibility, these mutants were examined for motility on the motility agar plates (Fig. 3). To our surprise, the results obtained were highly complicated. After incubation at $37^{\circ} \mathrm{C}$ for $5 \mathrm{~h}$, mutants defective in either one of the genes in the operon showed a motile phenotype; that is, they formed swarms on motility agar plates. The $f i D$ mutant (KK1391) formed minute swarms, while the fitT mutant (KK1393) formed swarms whose size was almost equivalent to, or somewhat smaller than, that of the swarms formed by the wild-type strain (KK1004). The fliS mutant (KK1392) formed swarms of an intermediate size. These results indicated that none of these genes are essential for swarm formation on the motility agar plates in S. typhimurium. Like the fiD: : Tn10 mutant (KK2601), the fiDS and fiDST mutants (KK1394 and KK1396) did not form swarms under these conditions. The fiST mutant (KK1395) formed swarms of almost identical size to those formed by the $f i S$ mutant.

\section{Flagellation of the fliD, flis and fliT mutants}

In order to examine flagellation with the $f i D$, fliS or $f i T$ mutants, cells of the individual mutants grown in L broth were negatively stained and observed by electron microscopy. As reported previously (Homma et al., 1984b), the fiD mutant (KK1391) did not produce filaments (data not shown). The reason why the fiD mutant formed ninute swarms on the motility agar plate is discussed later. Interestingly, the fiiS mutant (KK1392) was found to produce flagella with filaments much shorter than those produced by the wild-type strain (KK1004) (Fig. 4). Distribution of filament length was compared between the wild-type and $f i S$-mutant strains (Fig. 5). In the wildtype cells, the length varied from 1 to 10 wave units with a mean value of 3.5 wave units. On the other hand, in the fiS mutant, the maximal length did not exceed 3 wave units and more than $60 \%$ of the filaments were shorter than 1 wave unit. Flagellar structures were isolated from the fiiS mutant and inspected by electron microscopy.
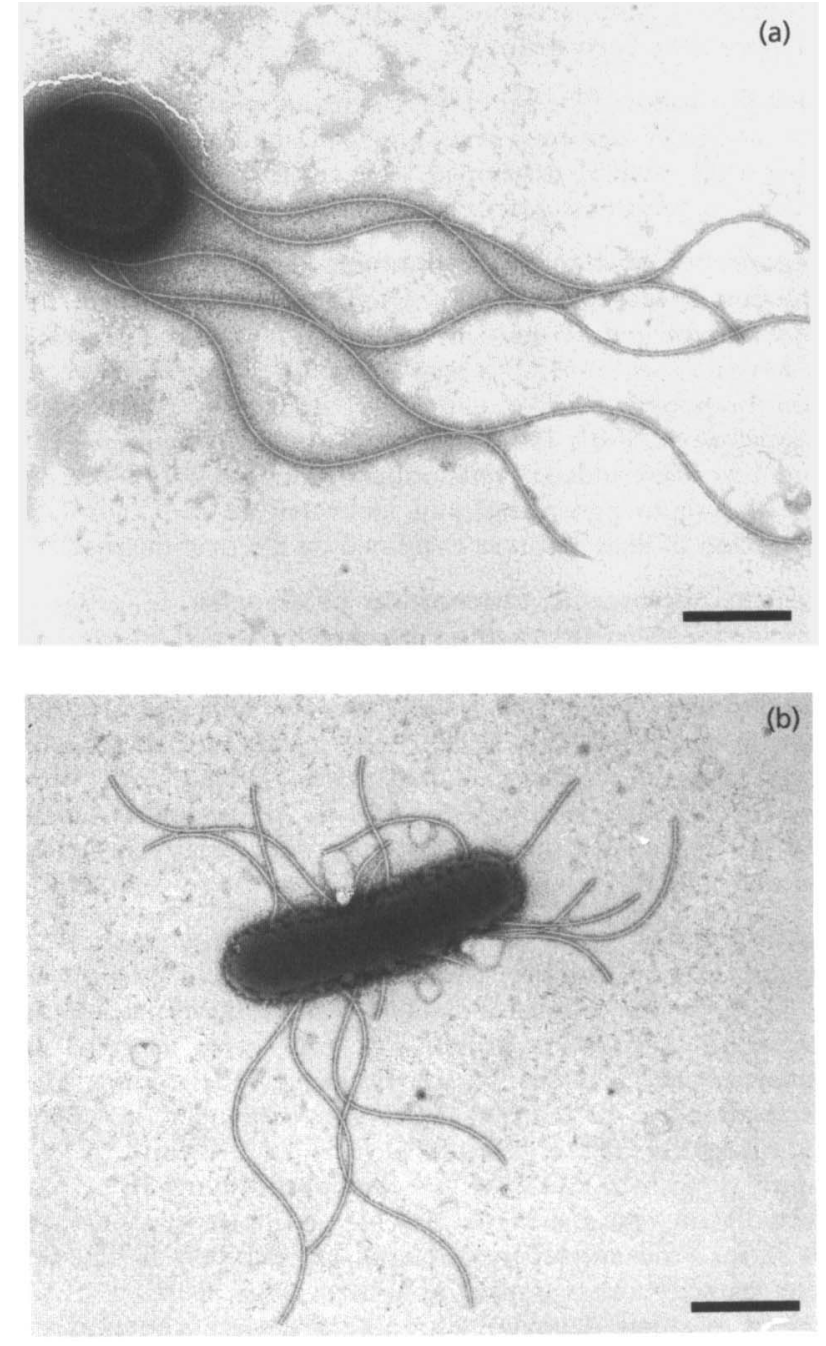

Fig. 4. Electron micrographs of cells of the wild-type (KK1004) (a) and flis-mutant KK1392 (b) strains. Cells grown in L broth were stained negatively with phosphotungstic acid and observed by electron microscopy. Micrographs were taken at a magnification of $\times 8000$. Bar, $1 \mu \mathrm{m}$. 


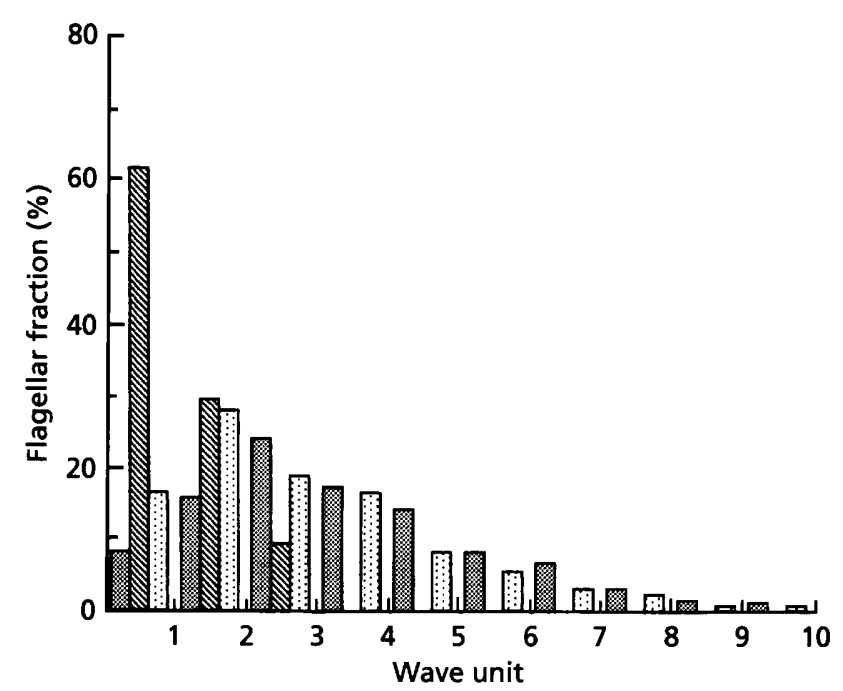

Fig. 5. Distribution of filament length in the wild-type (图), flis-

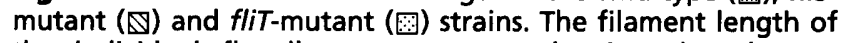
the individual flagellum was measured using the electron micrographs of cells stained negatively with phosphotungstic acid. Strains used ( $n=$ total number of flagella observed): wildtype, KK1004 $(n=180)$; flis mutant, KK1392 $(n=215) ;$ fliT mutant, KK1393 ( $n=133)$.

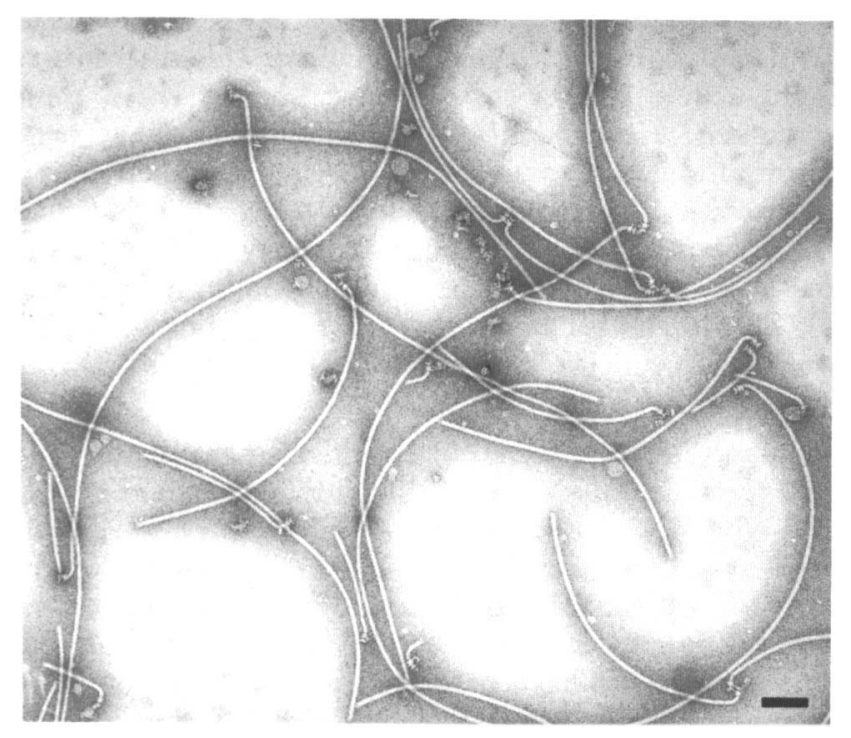

Fig. 6. Electron micrograph of flagellar structures produced by the flis mutant. Flagellar structures were fractionated from the cells of KK1392 by the method of Suzuki et al. (1978). The fractionated materials were negatively stained with phosphotungstic acid and observed by electron microscopy. The micrograph was taken at a magnification of $\times 20000$. Bar, $200 \mathrm{~nm}$.

Almost all of the hook-basal-body structures were found to have filament portions (Fig. 6), indicating that the fis mutation affects the elongation process but not the initiation process of filament assembly. Because the $f i T$ mutant constructed above (KK1393) carries a total deletion in the fitT gene, it should manifest a null phenotype for the fiT gene. Because we could not detect any obvious difference in flagellar structure and in filament length between the wild-type and $f i T$-mutant strains, it is unlikely that Fli'T has a direct role in flagellar formation and function. Therefore, we did not analyse further the fit mutant in this study.

\section{Complementation analysis}

The fis mutant constructed above (KK1392) retains codons 1-71 of the fis gene. Therefore, it might be possible that the small swarm formed by the fis mutant should be attributed to the truncated FliS protein. In order to exclude this possibility, plasmid pKKD4 was introduced by transformation into the fiDST mutant (KK1396) in which the entire fiS gene has been deleted, and motility recovery of the resulting transformant was examined. Because pKKD4 carried an intact $f i D$ gene and only the first eight codons of the fiS gene, the transformant was expected to show the null phenotype for the fis gene. The transformant was found to form small swarms on motility agar plates just like the fiS mutant (data not shown). Therefore, we conclude that formation of small swarms is the null phenotype for the fiiS gene.

\section{Excretion of flagellin by the flis mutant}

To find out why the fiS mutant produces short filaments, we tested the possibility that the fiiS mutation might affect the export process of flagellin. Export of flagellin was examined in the $f i D$-mutant background because the $f i D$ mutation is known to block filament assembly, resulting in unassembled flagellin molecules being excreted into the culture media (Homma et al., 1984a). Culture supernatants from the $f i D$ and $f i D S$ mutants (KK1391 and KK1394) were concentrated and analysed by SDS-PAGE (Fig. 7a). It was found that the amount of excreted flagellin is much smaller in the fiDS mutant than in the fiD mutant. In order to exclude the possibility that this difference might be caused by the difference in the amount of flagellin synthesized, total flagellin was analysed by Western blotting with whole cultures from the $f i D$ and $f i D S$ mutants (Fig. 7b). The amount of flagellin produced by the fiDS mutant was found to be almost identical to that produced by the $f i D$ mutant. This indicates that the $f i S$ mutation does not affect the process of flagellin synthesis. Therefore, we conclude that the fiS mutation affects the export process of flagellin.

In order to confirm our conclusion, we examined the in vitro reconstitution of filaments onto the hooks with exogenously supplied flagellin monomers under FliSdepletion conditions. Flagellin monomers used were purified either from the filaments produced by the wildtype strain (KK1004) or from those produced by the fis mutant (KK1392). Because exogenously supplied flagellin is known to polymerize onto the hook only in the absence of the FliD protein (Kagawa et al., 1983), we examined the efficiency of reconstruction of filaments onto the hooks of 
12

(a)

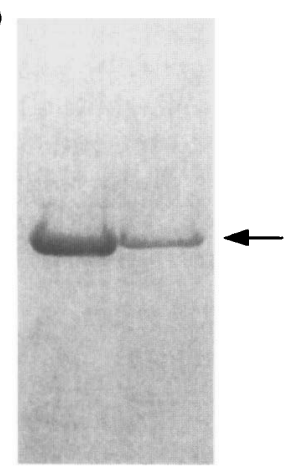

(b)

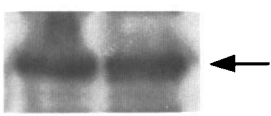

Fig. 7. Electrophoretic analysis of flagellin produced by the fliD and fliDS mutants. The arrows indicate the position of flagellin. Strains used: lane 1, fliD mutant (KK1391); lane 2, fliDS mutant (KK1394). (a) SDS-PAGE of culture supernatants. Proteins in the culture supernatants were precipitated with TCA and separated by SDS-PAGE. The gel was stained with Coomassie brilliant blue. (b) Immunological detection of flagellin in the whole cultures. Aliquots of liquid cultures containing a constant number of cells were separated by SDS-PAGE. The proteins on the gel were transferred onto a nitrocellulose membrane and subjected to Western blot analysis with a polyclonal antibody against flagellin.

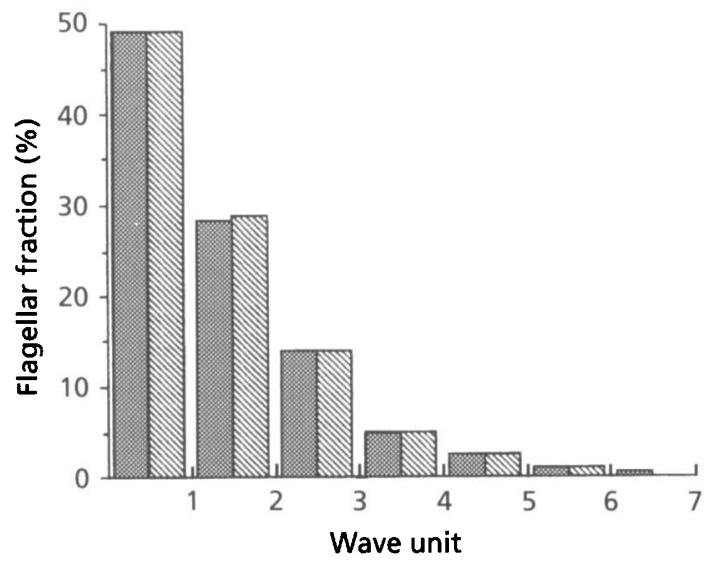

Fig. 8. Length distribution of filaments reconstituted onto the

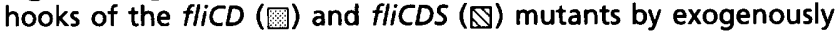
supplied flagellin monomers. The fliCD and fliCDS mutants were constructed by introducing the flic: :Tn 10 mutation by P22-mediated transduction from KK2604 to KK1391 and KK1394, respectively. The cells of the resulting mutants were incubated with flagellin monomers prepared from the wildtype strain KK1004. After incubation for $12 \mathrm{~h}$ at $26^{\circ} \mathrm{C}$, the cells were negatively stained with phosphotungstic acid and observed by electron microscopy. Total number of reconstituted filaments observed: fliCD mutant, 239; fliCDS mutant, 202.

the $f i D$ mutant. It was found that flagellin monomers from the fliS mutant polymerized with the same efficiency as those from the wild-type strain (data not shown). Next, we examined the reconstruction of filaments onto the hooks of the $f i D S$ mutant with the wild-type flagellin monomers (Fig. 7). In this experiment, the $f i C$ mutants were used to prevent endogenous flagellin monomers polymerizing onto the hooks. The reconstituted filaments in the fiCDS mutant were found to be as long as those in the fiiCD mutant. These results indicate that the fis mutation does not affect the polymerization efficiency of the flagellin molecule and that the exogenously supplied flagellin can polymerize in the absence of FliS as efficiently as in the presence of FliS.

\section{DISCUSSION}

In this study, we constructed mutants in which the individual genes in the $f i D$ operon on the S. typhimurium chromosome were specifically disrupted. Examination of flagellation with these mutants revealed that the fis and fiT genes were both dispensable for flagellar formation and function. This result is quite inconsistent with that in E. coli reported by Kawagishi $e t$ al. (1992). They reported that introduction of plasmids carrying the fiD gene alone or the fiDST regions with the fliS and fiT genes independently inactivated did not recover the motility of an E. coli strain with the chromosomal fiDST region deleted. Based on this observation, they concluded that the $f i S$ and $f i T$ genes are both indispensable for flagellation in E. coli. Chen \& Helmann (1994) reported evidence suggesting that the fis gene may be also essential for flagellation in $B$. subtilis. Of course, this discrepancy could reflect a species difference among these three organisms. However, because no difference has been reported in gene requirement for flagellation between $S$. typhimurium and E. coli (Kutsukake et al., 1980; Macnab, 1992), we anticipate that our result may be true also at least in E. coli. We suspect that the inability of the E. coli fiDST mutant to flagellate even in the presence of a plasmid carrying the $f i D$ gene might be due to a multicopy effect of the $f i D$ gene.

Although the fiiS gene is not essential for flagellation, the flis mutant produces much shorter filaments than the wild-type strain. Because almost all of the hook-basalbody structures produced by the fiiS mutant had filament portions, the fiiS mutation should affect the elongation step but not the initiation step of filament assembly. So far, several genes have been identified to be involved in the initiation process of filament assembly. They include $f g K, f g L, f i D$ and $f g N$ (Homma et al., 1984b; Kutsukake et al., 1994). We believe that fiS is the first documented gene whose mutation affects the elongation step of filament assembly. In the $f i D$-mutant background where flagellin molecules cannot assemble onto the hooks resulting in their excretion into the culture medium, the amount of flagellin excreted into the medium was shown to be much reduced by the $f i S$ mutation. Because the $f i S$ mutation does not affect the synthesis of flagellin, we conclude that the formation of short filaments in the fis mutant is attributed to impaired flagellin transport. Ikeda et al. (1993) reported that a fliD: : Tn10 mutant produces short filaments when supplied exogenously with purified FliD. This is consistent with our conclusion because the 
fiD : : $\operatorname{Tn} 10$ mutant is expected to be defective not only in $f i D$ but also in fiS owing to a polar effect of $\operatorname{Tn} 10$ insertion.

Flagellin molecules have been postulated to be exported to the growing tips through the channel residing in the pre-existing flagellar structures (Kuwajima et al., 1989). Namba et al. (1989) showed that the filament has a small hole through which flagellin monomers could be transported in an unfolded and stretched conformation. Using temperature-sensitive flagellation mutants of $S$. typhimurium, Vogler et al. (1991) identified some candidate genes involved in the export process of flagellin. They include $f h A, f i H, f i I$ and $f i N$. Although their exact roles have remained unknown, it has been postulated that they may constitute the flagellum-specific exposi apparatus at the cytoplasmic face of the basal body. What is the role of FliS in this export pathway? It has been suggested that FliS may be neither the structural component of flagellar structure nor an integral membrane protein (Kawagishi et al., 1992). Therefore, it may be localized in the cytosol. It is well known that several cytoplasmic chaperones, such as SecB, DnaK, and GroEL, are involved in the general pathway of protein secretion (Kumamoto, 1989; Phillips \& Silhavy, 1990). They bind to presecretory proteins to inhibit their folding and to pass them on to the secretion apparatus (Hartl et al., 1990). By analogy with this, we would like to propose a hypothesis that FliS may be a cytoplasmic chaperone specific for flagellin. FliS may bind to nascent flagellin molecules to maintain them in an unfolded state and to target them to the flagellum-specific export apparatus. In order to test this hypothesis, we are currently performing a biochemical analysis of the fis gene product.

In this study, we found that the $f i D$ mutant does not product filaments in liquid medium but does form minute swarms on motility agar plates. It has been discovered that the hook structures formed by the fiD mutant do not support the polymerization of endogenously supplied flagellin molecules, but act as the polymerization nuclei for exogenously supplied flagellin molecules (Homma et al., 1986). Because the unassembled flagellin molecules have been shown to be excreted into the medium (Homma et al., 1984a), we suspect that in the motility agar plates the excreted flagellin molecules could not diffuse freely into the media and accumulated around the cells resulting in them being assembled into filaments onto the hooks deficient in the FliD protein. Inability of the $f i D:: \operatorname{Tn} 10$, fiDS and fiDST mutants to form swarms on motility agar plates might reflect the impaired excretion of flagellin into the medium owing to the $f i S$ deficiency. Consistent with this, prolonged incubation or supplement of flagellin monomers into the motility agar plates could facilitate swarm formation by these mutants (our unpublished results).

Because a $f i D:: \operatorname{Tn} 10$ mutation causes over-expression of the flagellar late operons, the $f i D$ operon has been postulated to contain a negative regulator gene, $r f A$ (Kutsukake et al., 1990). The expression of the flagellar late operons is negatively controlled by the flagellumspecific anti-sigma factor, FlgM (Ohnishi et al., 1992), whose intracellular activity is regulated by being excreted through the flagellar structure (Hughes et al., 1993; Kutsukake, 1994). We showed that the secretion of FlgM is enhanced by the $f i D:: \operatorname{Tn} 10$ mutation and proposed that the $\operatorname{rfl} A$ function of the $f i D$ operon may be attributed to the flagellar cap protein encoded by the $f i D$ gene (Kutsukake, 1994). However, at present, we cannot exclude the possibility that the fis or fliT gene may correspond to the $r f A$ gene. This problem will be solved by analysing the expression levels of the late operons and excretion levels of FlgM in the defined mutants in the $f i D$ operon constructed in the present study.

The flagellar regulon of $S$. typhimurium includes more than 50 genes (Kutsukake et al., 1990). Among them, three genes, $f i L, f h E$ and $f i T$, have been reported to be totally dispensable for flagellar formation and function (Raha et al., 1994; Minamino et al., 1994; this study). At present, we have no idea of the roles of these genes. More careful examination of the structure and function of flagella with the corresponding mutants may be required for understanding their roles in flagellar morphogenesis and function.

\section{ACKNOWLEDGEMENTS}

Electron microscope facilities were provided by the Center for Gene Science, Hiroshima University. This work was supported by a Grant-in-Aid for Scientific Research from the Ministry of Education, Science and Culture, Japan.

\section{REFERENCES}

Asakura, S., Eguchi, G. \& lino, T. (1964). Reconstitution of bacteria flagella in vitro. $J \mathrm{Mol}$ Biol 10, 42-56.

Chen, L. \& Helmann, J. D. (1994). The Bacillus subtilis sigma ${ }^{D}$ dependent operon encoding the flagellar proteins FliD, FliS, and FliT. J Bacteriol 176, 3093-3101.

Hartl, F.-U., Lecker, S., Schiebel, E., Hendrick, J. P. \& Wickner, W. (1990). The binding cascade of SecB to SecA to SecY/E mediates preprotein targeting to the E. coli plasma membrane. Cell 63, 269-279.

Homma, M. \& lino, T. (1985). Locations of hook-associated proteins in flagellar structures of Salmonella typhimurium. J Bacteriol 162, 183-189.

Homma, M., Fujita, H., Yamaguchi, S. \& lino, T. (1984a). Excretion of unassembled flagellin by Salmonella typhimurium mutants deficient in hook-associated proteins. J Bacteriol 159, 1056-1059.

Homma, M., Kutsukake, K., lino, T. \& Yamaguchi, S. (1984b). Hook-associated proteins essential for flagellar filament formation in Salmonella typhimurium. J Bacteriol 157, 100-108.

Homma, M., lino, T., Kutsukake, K. \& Yamaguchi, S. (1986). In vitro reconstitution of flagellar filaments onto hooks of filamentless mutants of Salmonella typhimurium by addition of hook-associated proteins. Proc Natl Acad Sci US A 83, 6169-6173.

Hughes, K. T., Gillen, K. L., Semon, M. J. \& Karlinsey, J. E. (1993). Sensing structural intermediates in bacterial flagellar assembly by export of a negative regulator. Science 262, 1277-1280.

Ikeda, T., Asakura, S. \& Kamiya, R. (1985). 'Cap' on the tip of Salmonella flagella. J Mol Biol 184, 735-737.

Ikeda, T., Homma, M., lino, T., Asakura, S. \& Kamiya, R. (1987). Localization and stoichiometry of hook-associated proteins within Salmonella typhimurium flagella. J Bacteriol 169, 1168-1173. 
Ikeda, T., Asakura, S. \& Kamiya, R. (1989). Total reconstruction of Salmonella flagellar filaments from hook and purified flagellin and hook-associated proteins. J Mol Biol 209, 109-114.

Ikeda, T., Yamaguchi, S. \& Hotani, H. (1993). Flagellar growth in a filament-less Salmonella fiD mutant supplemented with purified hook-associated protein 2. J Biochem 114, 39-44.

Kagawa, H., Morishita, H. \& Enomoto, M. (1981). Reconstruction in vitro of flagellar filaments onto hook structures attached to bacterial cells. $J$ Mol Biol 153, 465-470.

Kagawa, H., Nishiyama, T. \& Yamaguchi, S. (1983). Motility development of Salmonella typhimurium cells with fla $\mathrm{V}$ mutations after addition of exogenous flagellin. J Bacteriol 155, 435-437.

Kawagishi, I., Muller, V., Williams, A. W., Irikura, V. M. \& Macnab, R. M. (1992). Subdivision of flagellar region III of the Escherichia coli and Salmonella typhimurium chromosomes and identification of two additional flagellar genes. J Gen Microbiol 138, 1051-1065.

Kumamoto, C. A. (1989). Escherichia coli SecB associates with exported protein precursors in vitro. Proc Natl Acad Sci USA 86, $5320-5324$.

Kutsukake, K. (1994). Excretion of the anti-sigma factor through a flagellar substructure couples flagellar gene expression with flagellar assembly in Salmonella typhimurium. Mol \& Gen Genet 243, 605-612.

Kutsukake, K., lino, T., Komeda, Y. \& Yamaguchi, S. (1980). Functional homology of $f a$ genes between Salmonella typhimurium and Escherichia coli. Mol \& Gen Genet 178, 59-67.

Kutsukake, K., Nakao, T. \& lino, T. (1985). A gene for DNA invertase and an invertible DNA in Escherichia coli K-12. Gene 34, 343-350.

Kutsukake, K., Ohya, Y., Yamaguchi, S. \& lino, T. (1988). Operon structure of flagellar genes in Salmonella typhimurium. Mol \& Gen Genet 214, 11-15.

Kutsukake, K., Ohya, Y. \& lino, T. (1990). Transcriptional analysis of the flagellar regulon of Salmonella typhimurium. J Bacteriol 172, $741-747$.

Kutsukake, K., Okada, T., Yokoseki, T. \& lino, T. (1994). Sequence analysis of the $\operatorname{fg} A$ gene and its adjacent region in Salmonella typhimurium, and identification of another flagellar gene, $f g N$. Gene 143, 49-54.

Kuwajima, G., Kawagishi, I., Homma, M., Asaka, J.-I., Kondo, E. \& Macnab, R. M. (1989). Export of the N-terminal fragment of
Escherichia coli flagellin by a flagellum-specific pathway. Proc Natl Acad Sci US A 86, 4953-4957.

Macnab, R. M. (1992). Genetics and biogenesis of bacterial flagella. Annu Rev Genet 26, 131-158.

Minamino, T., lino, T. \& Kutsukake, K. (1994). Molecular characterization of the Salmonella typhimurium $f h B$ operon and its protein products. $J$ Bacteriol 176, 7630-7637.

Namba, K., Yamashita, I. \& Vonderviszt, F. (1989). Structure of the core and central channel of bacterial flagella. Nature 342, 648-654.

Ohnishi, K., Kutsukake, K., Suzuki, H. \& lino, T. (1992). A novel transcriptional regulation mechanism in the flagellar regulon of Salmonella typhimurium: an anti-sigma factor inhibits the activity of the flagellum-specific sigma factor, $\sigma^{\mathbf{F}}$. Mol Micrabiol 6, $3149-3157$.

Phillips, G. J. \& Silhavy, T. J. (1990). Heat-shock proteins DnaK and GroEL facilitate export of LacZ hybrid proteins in E. coli. Nature 344, 882-884.

Raha, M., Sockett, H. \& Macnab, R. M. (1994). Characterization of the fiL gene in the flagellar regulon of Escherichia coli and Salmonella typhimurium. J Bacteriol 176, 2308-2311.

Sanger, F., Nicklen, S. \& Coulson, A. R. (1977). DNA sequencing with chain-terminating inhibitors. Proc Natl Acad Sci USA 74, 5463-5467.

Suzuki, T., lino, T., Horiguchi, T. \& Yamaguchi, S. (1978). Incomplete flagellar structures in nonflagellate mutants of Salmonella typhimurium. J Bacteriol 133, 904-915.

Takeshita, S., Sato, M., Toda, M., Masahashi, W. \& HashimotoGotoh, T. (1987). High-copy-number and low-copy-number plasmid vectors for lac $Z$ alpha-complementation and chloramphenicol- or kanamycin-resistance selection. Gene 61, 63-74.

Vogler, A. P., Homma, M., Irikura, V. M. \& Macnab, R. M. (1991). Salmonella typhimurium mutants defective in flagellar filament regrowth and sequence similarity of FliI to $\mathrm{F}_{0} \mathrm{~F}_{1}$, vacuolar, and archaebacterial ATPase subunits. J Bacteriol 173, 3564-3572.

Yamada, M., Hakura, A., Sofuni, T. \& Nohmi, T. (1993). New method for gene disruption in Salmonella typhimurium: construction and characterization of an ada-deletion derivative of Salmonella typhimurium TA1535. J Bacteriol 175, 5539-5547.

Received 6 March 1995; accepted 15 March 1995. 